\title{
Bone Marrow Morphology in Myeloproliferative Neoplasms - Relevance in the Molecular Era
}

\author{
Suchi Ghai, Sharada Rai*, Cheryl Sarah Philipose and Sonia Kaur Singh \\ Department of Pathology, Kasturba Medical College, (Manipal Academy of Higher education) Mangalore, Karnataka, India
}

\section{ABSTRACT}

Background: Myeloproliferative neoplasms (MPNs) are clonal hematopoietic disorders. The mutation BCR-ABL is sine qua non for diagnosis of chronic myeloid leukemia and JAK2 V617F is present in majority of cases of polycythemia vera (PV), essential thrombocythemia (ET) and primary myelofibrosis (MF). Recent 2016 classification consigns importance to BM morphology as mutations are not universal. Identification of specific features would aid diagnosis of JAK2V617F negative MPN. This study aims to assess utility of peripheral blood and bone marrow morphology in myeloproliferative neoplasms in correlation with BCR-ABL and JAK2V617F.

Methods: A retrospective 7 years study of peripheral blood, bone marrow aspirate (BMA) and bone marrow biopsy (BMB) of 44 cases of myeloproliferative neoplasms with BCR-ABL and JAK2 V617F mutation analysis.

Results: Forty four cases of MPNs including 24 (54.5\%) cases of CML, 7 (15.9\%) cases of PV, 5 (11.3\%) cases of MF, 3 (6.8\%) cases of ET, and $5(11.3 \%)$ cases of MPN-U. MPV and LDH were significantly higher in MF in comparison to PV and ET. Higher haemoglobin (Hb) and platelets were seen in PV and ET. Dwarf MKs with hypolobated, cloud like nuclei in CML (fig 1), large MKs with staghorn nuclei were seen in ET. normal sized MKs with cloud like hypolobated forms in PV(fig2) and cloud like atypical dysmorphic nuclei with dense clustering were seen in MF.

Conclusion: Hb, mean platelet volume (MPV), and LDH in conjunction with MK morphology aids in the diagnosis of MPNs. These findings will enable accurate diagnosis in JAK2 negative MPNs and benefit low resource center.

Keywords: Myeloproliferative Neoplasm, LDH, Bone Marrow.

\section{Introduction}

Myeloproliferative neoplasms (MPN) are group of clonal disorders of hematopoietic stem cell origin. The newer classification system of MPN incorporated mutations discovered in the JAK2 and MPL genes. Newer genetic mutations like CALR and CSF3R are also being associated with MPNs. ${ }^{[2,3,4,5]}$

Somatic mutation in JAK2 was related to the three disease entities in MPNs. ${ }^{[3,4,5]}$ Morphology of MKs has been emphasized in literature in the diagnosis of these conditions, but confusion pervades regarding terminologies used in their description. This results in underutilization of BM morphology and increasing reliance on genetic markers.

This study is an attempt to study the peripheral blood and $\mathrm{BM}$ and identify specific MK features in the subcategories of MPNs in correlation with BCR-ABL and JAK2 V617F mutation. The main focus of our study was on MK number, morphological types, pattern, localization, along with other hematopoietic cells, in BM along with clinical features, $\mathrm{Hb}$, WBC count, platelet count, MPV, PDW and LDH. These features were correlated with final diagnosis

\section{Materials and Methods}

This was a retrospective and prospective hospital based study, from January 2011 to December 2017 including review of clinical presentation, peripheral blood, BMA, and BMB along with clinical features in cases with BCR$\mathrm{ABL}$ and JAK2 V617F mutation studies. The study was conducted in tertiary care centre. A total of 44 cases of MPN with genetic studies were collected during this time period. The peripheral smear and BMA smears were stained by Leishman stain. BMBs were stained by routine hematoxylin and eosin (H\&E). Reticulin staining was performed by Gomori's method and graded using WHO guidelines. ${ }^{[2]}$

The peripheral blood, BMA and BMB slides were studied with emphasis on megakaryocytic morphology and pattern. MK morphology definitions were considered for the study. ${ }^{[4]}$

Proportions, graphs and tables were used to analyze data. Chi-square value $\left(\mathrm{c}^{2}\right)$ and Fischer test, were applied as appropriate. $\mathrm{P}$ value of $<0.05$ was considered significant. Analysis was done using SPSS version 17.0 .Clearance from institutional ethics committee was taken. 


\section{Result}

A total of 44 cases diagnosed as MPN on complete blood count, peripheral blood examination, BMA and BMB with cytogenetic mutation study were included. This constituted 24 cases $(54.4 \%)$ of chronic myeloid leukemia, 7 cases (46.6\%) of PV, 3 cases $(6.8 \%)$ of ET, 5 cases $(11.4 \%)$ were of MF and 5 cases $(11.4 \%)$ of myeloproliferative neoplasm-unclassified.

The commonest age group affected was between 40 to 60 years in all disease categories except MPN-U where majority of cases were under 40 years of age. Males were affected predominantly in all the five disease entities with M: F ratio of 1.1:1 in CML, 6:1 in PV, 2:1 in ET, 3:2 in MF and 4:1 in MPN-U.

Weakness was the most common symptom in CML (95.8\%) in 22 out of 24 cases, 6 out of 7 cases of PV (85.7\%), one case of ET (33.3\%), all 5 cases of MF (100\%) and 4 out of 5 cases $(80 \%)$ of MPN-U. The patients of PV also presented with plethora in 6 out of 7 cases $(85.7 \%)$. Thrombotic and bleeding episodes were noted in $4.5 \%$ cases of PV and ET respectively. Fatigue, abdominal pain, fever, weight loss, palpitation, and giddiness were other overlapping features. Splenomegaly was the most common physical sign (100\%) among all disease

The hemoglobin, total white cell count and platelet parameters are depicted in Table 1. Comparison of MPV showed lower values in PV compared prefibrotic MF. PDW showed similar values for all the entities of MPN (table 2). The LDH and serum uric acid is given in Table 3.

Significant peripheral smear findings included red cell crowding in all cases $(100 \%)$ of PV cases. Basophils were present in all the cases of MPN in peripheral smears studied.

In BMA and BMB increased cellularity and panmyelosis was seen in all cases $(100 \%)$ of PV, ET, MPN-U. Myelofibrosis revealed normal to increased cellularity in 3 cases $(60 \%)$ and decreased cellularity in 2 cases ( $40 \%)$. Dry tap was noted in 2 cases $(40 \%)$. The morphology of MKs in bone marrow in the different categories is enlisted in Table 4, 5. The $\mathrm{p}$ value obtained in all the features of MK morphology was found to be less than 0.05 . Reticulin grading in different MPNs is shown Table 6.

TABLE 1: Mean and range of Haemoglobin, total RBC count, total WBC count and platelet count in MPNs.

\begin{tabular}{|c|c|c|c|c|}
\hline Diagnosis & $\begin{array}{c}\text { Hemoglobin } \\
\text { Mean (range) (g/dl) }\end{array}$ & $\begin{array}{l}\text { Total RBC count } \\
\left(\times 10^{12} / L\right)\end{array}$ & $\begin{array}{c}\text { Total WBC count } \\
\left(\times 10^{9} / \mathrm{L}\right)\end{array}$ & $\begin{array}{l}\text { Total platelet count } \\
\text { (x 10\%) }\end{array}$ \\
\hline $\mathrm{CML}$ & $9.2(5-12.5)$ & $3.2(1.70-5.04)$ & $157.50(3-506)$ & $3.50(0.13-15.6)$ \\
\hline PV & $18.9(18.4-19.8)$ & $6.8(5.25-8.12)$ & $14.81(8-21)$ & $4.26(2.14-8.06)$ \\
\hline ET & $12.9(11.1-15.6)$ & $5.4(4.56-6.64)$ & $20.67(11-28)$ & $8.54(6.08-12.38)$ \\
\hline MF & $11.8(11-13.1)$ & $5.07(3.86-6.94)$ & $32.94(6-55)$ & $5.19(0.30-8.62)$ \\
\hline MPN-U & $11.6(6.5-16.4)$ & $5.40(3.15-6.99)$ & $18.70(10-36)$ & $7.20(2.46-11.49)$ \\
\hline
\end{tabular}

(CML- chronic myeloid leukemia, PV - Polycythemia vera, ET-Essential thrombocythemia and MF-Myelofibrosis and MPN-U-Myeloproliferative neoplasmUnclassified)

Table 2: Comparison of Mean platelet volume and Platelet distribution width in various entities of Myeloproliferative neoplasms.

\begin{tabular}{|c|c|c|}
\hline Diagnosis & MPV(fL) & PDW (\%) \\
\hline CML & $9.10(7.2-13.3)$ & $18.57(9-19.9)$ \\
\hline PV & $8.55(7.1-10.2)$ & $18.5(18.3-18)$ \\
\hline ET & $8.7(8.5-8.9)$ & $18(17-19.1)$ \\
\hline MF & $9.5(8.5-11.3)$ & $18.5(18.6-19)$ \\
\hline MPN-U & $9.4(7.3-10.4)$ & \\
\hline
\end{tabular}

TABLE 3: Mean value and range of LDH and Uric acid in MPNs.

\begin{tabular}{|c|c|c|}
\hline Diagnosis & LDH Mean (range) (U/L) & Uric acid Mean (range) (mg/dl) \\
\hline CML & $1115(9309-2420)$ & $6.13(2.4-7.8)$ \\
\hline PV & $577.7(379-796)$ & $8.2(6.2-9.8)$ \\
\hline ET & $487(476-498)$ & $6.2(4.9-5.5)$ \\
\hline MF & $1016(1000-1030)$ & $6.5(6.2-6.8)$ \\
\hline MPN - U & $432(304-560)$ & $4.75(4.5-5)$ \\
\hline
\end{tabular}


TABLE 4: Comparison of Megakaryocytic number and size in bone marrow biopsy in PV, ET and MF and MPN-U. (P value < 0.05 for size chi square test).

\begin{tabular}{|c|c|c|c|c|c|c|}
\hline \multicolumn{2}{|c|}{ Features } & CML & PV & ET & MF & MPN-U \\
\hline \multirow{3}{*}{ Number } & Normal & $45.8 \%$ & $0 \%$ & $0 \%$ & $20 \%$ & $0 \%$ \\
\hline & Increased & $50 \%$ & $100 \%$ & $100 \%$ & $80 \%$ & $100 \%$ \\
\hline & Decreased & $4.2 \%$ & $0 \%$ & $0 \%$ & $0 \%$ & $0 \%$ \\
\hline \multirow{3}{*}{ Size } & Normal & $0 \%$ & $100 \%$ & $0 \%$ & $100 \%$ & $100 \%$ \\
\hline & Small & $95.8 \%$ & $0 \%$ & $0 \%$ & $0 \%$ & $0 \%$ \\
\hline & Large & $4.2 \%$ & $0 \%$ & $100 \%$ & $0 \%$ & $0 \%$ \\
\hline \multirow{3}{*}{ Cytoplasm } & Normal & $4.2 \%$ & $0 \%$ & $33.3 \%$ & $0 \%$ & $0 \%$ \\
\hline & Small & $91.7 \%$ & $85.7 \%$ & $0 \%$ & $100 \%$ & $100 \%$ \\
\hline & Large & $4.2 \%$ & $14.3 \%$ & $66.7 \%$ & $0 \%$ & $0 \%$ \\
\hline
\end{tabular}

TABLE 5: Comparison of Megakaryocytic nuclear features and clustering in subcategories of MPNs . (P value $<0.05$ for nuclei, lobation, clustering, chi square test).

\begin{tabular}{|c|c|c|c|c|c|c|}
\hline \multicolumn{2}{|c|}{ Features } & CML & PV & ET & MF & MPN-U \\
\hline \multirow{4}{*}{ Nuclei } & Staghorn & $4.3 \%$ & $4.2 \%$ & $66.3 \%$ & $20 \%$ & $40 \%$ \\
\hline & Cloud like & $79.1 \%$ & $78.2 \%$ & $33.7 \%$ & $60 \%$ & $60 \%$ \\
\hline & Dysmorphic & $0 \%$ & $6.3 \%$ & $0 \%$ & $8.3 \%$ & $0 \%$ \\
\hline & Bare nuclei & $8.3 \%$ & $8.3 \%$ & $0 \%$ & $20 \%$ & $0 \%$ \\
\hline \multirow{3}{*}{ Lobation } & Normal & $0 \%$ & $0 \%$ & $0 \%$ & $0 \%$ & $0 \%$ \\
\hline & Hyperlobation & $16.7 \%$ & $14.3 \%$ & $100 \%$ & $20 \%$ & $40 \%$ \\
\hline & Hypolobation & $82.3 \%$ & $85.7 \%$ & $0 \%$ & $80 \%$ & $60 \%$ \\
\hline \multirow{3}{*}{ Clustering } & No & $4.2 \%$ & $0 \%$ & $0 \%$ & $0 \%$ & $0 \%$ \\
\hline & Loose & $91.7 \%$ & $41.9 \%$ & $33.7 \%$ & $40 \%$ & $60 \%$ \\
\hline & Dense & $8.3 \%$ & $58.1 \%$ & $66.7 \%$ & $60 \%$ & $40 \%$ \\
\hline
\end{tabular}

TABLE 6: Comparison of reticulin stain in BMB in subcategories of MPNs (P value $<0.05$ for reticulin).

\begin{tabular}{|c|c|c|c|c|c|c|}
\hline \multicolumn{2}{|c|}{ Features } & CML & PV & ET & MF & MPN-U \\
\hline \multirow{3}{*}{ Reticulin } & 0 & $4.2 \%$ & $14.3 \%$ & $0 \%$ & $0 \%$ & $0 \%$ \\
\cline { 2 - 7 } & 1 & $87.5 \%$ & $71.4 \%$ & $100 \%$ & $80 \%$ \\
\cline { 2 - 7 } & 2 & $8.3 \%$ & $14.3 \%$ & $0 \%$ & $80 \%$ & $20 \%$ \\
\hline
\end{tabular}

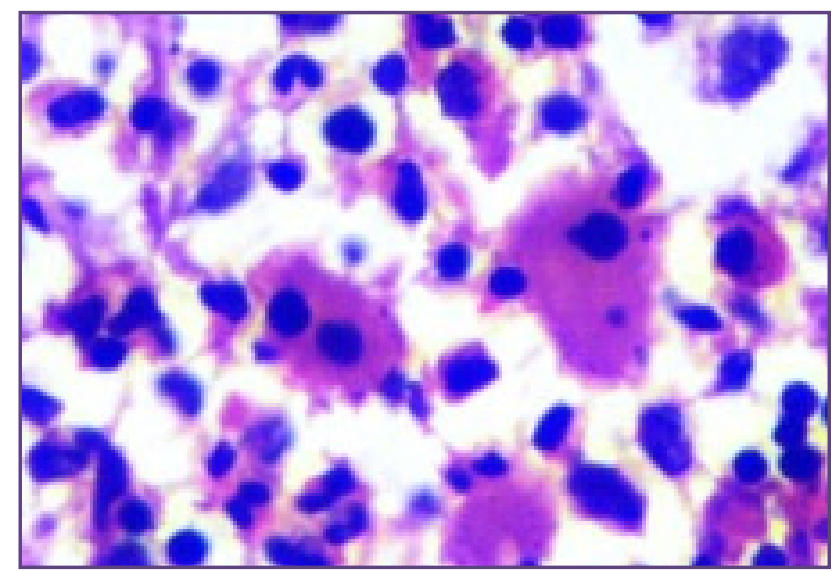

Fig. 1: Dwarf hypolobated MKs in Chronic myeloid neoplasm, Leishman stain X 400 .

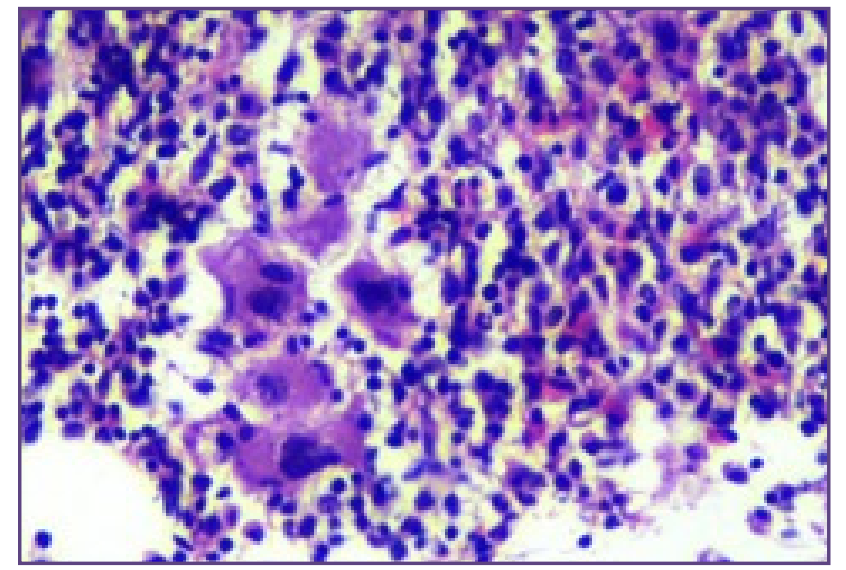

Fig. 2: Cloud like, hypolobated nuclei of MKs, Leishman stain X 400. 


\section{Discussion}

Transformation of hematopoietic stem cell forms the main essence of myeloproliferative neoplasms. ${ }^{[5,6]}$ The different entities included under MPN have cardinal morphologic features which help in distinguishing them from each other. Despite these differences these disorders share many common attributes too. Hence, it is imperative to recognize features which can help in distinguishing them from one another. Our study of MPNs assessed clinical, biochemical and $\mathrm{BM}$ changes with emphasis on the morphology of MKs specific changes in MPNs.

In our study CMLs, commonly presented with weakness (23 out of 24 cases, $95.8 \%$ ) and splenomegaly ( 21 out of 24 cases, $87.5 \%$ ). Jameel et al ${ }^{[6]}$ found splenomegaly in $98 \%$ of cases. The MPV value was found to be normal for the cases of CML and a high normal value was noted for PDW. Similar findings were reported in studies done by other authors ${ }^{[7,8]}$ High LDH values (mean $1115 \mathrm{u} / \mathrm{L}$ ) were noted in comparison to other MPNs. The marginal increase in the PDW value is contributed by an increase in both small and large platelets. ${ }^{[8]}$ We observed dwarf MKs with cloud like nuclei and hypolobation (Fig 1). Schelkar et al ${ }^{\left[{ }^{[]}\right.}$ provided evidence regarding morphological differences in MKs based on ultrastructural measurement and dwarf MKs were not seen in any other categories of MPN except CML

Presence of myelocytes, increased number of segmented neutrophils, eosinophilia and basophilia seen in peripheral smear as well as both BMA and BMBs in all the cases, further support the diagnosis of CML in comparison to PV, ET, MF and MPN-U.

Cases of PV, ET and MF in our study exhibited unique clinical, blood and BM highlights along with other overlapping features. All cases of MPNs presented with weakness and splenomegaly, patients of PV presented with thrombotic events and ET predominated with bleeding episodes. The mean $\mathrm{Hb}$ value $(18.9 \mathrm{~g} / \mathrm{dl})$ and total $\mathrm{RBC}$ count $\left(6.8 \times 10^{9} / \mathrm{L}\right)$ was higher in PV compared to other categories of MPN. Mean total WBC count was the highest in MF $\left(32.94 \times 10^{9} / \mathrm{L}\right)$ followed by ET $\left(20.67 \times 10^{\%} / \mathrm{L}\right)$ and PV $\left(14.81 \times 10^{9} / \mathrm{L}\right)$.

Platelet count was the highest in ET $\left(8.54 \times 10^{9} / \mathrm{L}\right)$ followed by MPN-U $\left(7.2 \times 10^{9} / \mathrm{L}\right), \mathrm{MF}\left(5.19 \times 10^{9} / \mathrm{L}\right)$. The mean value of MPV in PV (mean 8.55fL) was akin to ET (mean 8.7) and lower than the corresponding values in MF ( mean 9.50). Hence MPV may be of value in differentiating PV from prefibrotic MF. However significant difference was not noted in the PDW among the different MPNs as described by other authors. ${ }^{[8,10]}$ MPV and PDW play a significant role in differentiating reactive thrombocytosis from ET as the former has low mean platelet volumes. ${ }^{[7]}$ Other studies have also found that PDW value in MPNs is higher due to heterogeneity of platelet size. ${ }^{[8,9,10]}$

Serum LDH values were noticeably lower in PV (mean 577.7U/L), ET (mean $487 \mathrm{U} / \mathrm{L}$ ) and MPN- U (mean 432 $\mathrm{U} / \mathrm{L}$ ) in comparison to MF (mean 1016U/L). Serum LDH values, twice the upper limit of normal has been found to reasonably good discriminator of ET and PV from MF by some authors though overlaps with other conditions can occur. $^{[11,12]}$

BMA and BMB exhibited trilineage hypercellularity in all MPNs except in cases of overt myelofibrosis (4.5\%) where the bone marrow yielded dry tap in BMAs and was hypocellular due to fibrosis. The MKs exhibited distinct features in the subtypes of MPNs. MKs in PV were normal sized with mature cytoplasm $(100 \%)$ with cloud like nuclei $(79.2 \%)$, hypolobation $(85.7 \%$ ) and arranged in both loose (57.1\%) and dense clusters( $42.9 \%)$. In contrast ET was predominantly characterized by large $(66.3 \%)$, hyperlobated MKs (100\%) with staghorn nuclei $(66.7 \%)$ in dense clusters as noted by some authors. Normal sized MKs $(100 \%)$ with hypolobated $(80 \%)$, cloud like $(60 \%)$ dysmorphic nuclei characterized MFs. These finding have been highlighted by few authors and deserve greater attention. ${ }^{[13,14,15]}$

Normal sized MKs with cloud like hypolobated nuclei were an overlapping feature of PV and MF. These similarities are probably attributed to the allele burden and disease presentation in JAK2 V617F mutation MPNs with increased chances for the MF to resemble PV with higher copy number of mutated JAK2 V617F mutation. . $^{[4,16,17,18]}$

Grade 1fibrosis was seen in $71.4 \%$ cases of PV, all cases $(100 \%)$ and $(80 \%)$ of MPN-U. In MF, (80\%) exhibited grade 2 fibrosis while in $20 \%$ grade 3 overt fibrosis was evident. These findings were similar to study done by Thiele et al ${ }^{[11]}$, Michiels et al ${ }^{[13,14]}$. Role of MKs in mediating marrow fibrosis has been recognized in MPNs. Tentative hypothesis include increased release by MKs of growth factors like TGF- $\beta$ and PDGF along with aberrant $P$ selectin distribution which induces fibroblasts to produce collagen. ${ }^{[12,19,20]}$ Increased production of lysyl oxide (LOX) by fibrogenic cells has also been implicated. LOX oxidizes the PDGF receptor and enhances proliferation of the low ploidy megakaryocytes. ${ }^{[21,22,23]}$

It is intriguing how a single mutation in JAK2V617 results in a spectrum of diseases of MPNs though microRNA methylation and other synergic mutations may play a role. It is often difficult to differentiate PV and ET from prefibrotic MF. ${ }^{[24,25,26]}$ Similarly it is may be challenging 
to differentiating post PV and post ET myelofibrosis. In other studies, the simultaneous presence of loose clusters of large MKs and nuclear hyperlobation was significantly associated with ET and not in the prefibrotic and fibrotic Myelofibrosis. They also noted the association of dense clusters of MKs with maturation defects and bulbous nuclei pointed to a diagnosis of MF as also seen in our study ${ }^{[27,28,29]}$ Hence recognizing these specific features will facilitate differentiation between PV,ET and MF and aid accurate diagnosis when mutations are not identified.

\section{Conclusion}

MPNs consist of a diverse group of clonal disorders. We found that MPV and LDH were significantly higher in MF in comparison to $\mathrm{PV}$ and $\mathrm{ET}$ while higher $\mathrm{Hb}$ and platelets were seen in PV and ET. MKs exhibited distinctive features. Dwarf MKs arranged in loose clusters with cloud like, hypolobated nuclei predominated in CML. PV exhibited, both dense and loose clusters of normal sized MKs with hypolobated cloud like nuclei. In contrast, giant mature MKs with staghorn, hyperlobated nuclei in dense clusters were noted in ET. Small to normal sized megakaryocytes with dysmorphic hypolobated nuclei were seen in dense clusters in MF. However, MPN-U showed overlapping features clinically and in BM with other MPNs. Hence, clinical and hematological findings along with characteristic megakaryocytic features can help arrive at an accurate diagnosis of MPNs and is relevant in the present era.

This study needs to be validated with the newer genetic mutations introduced in the 2016 classification of MPNs in a larger patient sample size.

\section{Reference}

1. Campbell PJ, Green AR. The myeloproliferative disorders. N Engl J Med 2006; 335:2452-66

2. Arber AD, Orazi A, Hasserjian R, Thiele J, Borowitz MJ, Le Beau MM et al. The 2016 revision to the World Health Organization (WHO) classification of myeloid neoplasms and acute leukemia. Blood 2016; 1:1-45

3. Swerdlow, S. H. et al. (eds). WHO classification of Tumours of Haematopoietic and Lymphoid Tissues. 4th edn, (International Agency for Research on Cancer, Lyon, France, 2017).

4. Koopmans SM, Bot FJ, Lam KH, Van Marion AMW, De Raeve H, Hebeda KM. Reproducibility of histologic classification in nonfibrotic myeloproliferative neoplasia. Am J Clin Pathol 2011;136:618-624.

5. Spivak JL, Silver RT. The revised World Health Organization diagnostic criteria for Polycythemia vera, Essential thrombocythemia, and Primary myelofibrosis: An alternative proposal. Blood 2008;112(2):231-9
6. Jameel A, Jamil SN. Clinico-pathological profile of chronic myeloid leukemia. JPMI 2006;20(3):235-238

7. Rumi, E, \& Cazzola, M. (2017). Diagnosis, risk stratification, and response evaluation in classical myeloproliferative neoplasms. Blood,129(6), 680-692.

8. Lelie Der JV, Borne Dem AK. Platelet volume analysis for differential diagnosis of thrombocytosis. J Clin Pathol 1986;39:129-133

9. Schelkar R, Florea A, Urean L, Patiu M, Petrov L. Differential diagnosis between chronic granulocytic leukemia, polycythemia vera and essential thrombocythemia using micro- and ultrastructural measurement data performed at the level of MKs. Romanian Journal of Morphology and Embryology 2009;50(4):595-603

10. Olteanu AL, Mihaila RG, Catana AC, Flucus O, Bus C, Mihalache M. Platelet indices in Philadelphia-negative chronic myeloproliferative neoplasms. Revista Romana de Medicina de Laborotor 2015;23(2):169-177

11. Thiele JM, Kvasnicka HM. Diagnosis of Polycythemia vera based on bone marrow pathology. Curr Hematol Rep 2005; 4(3):218-23

12. Philip AB, Campbell PJ , Green AR. Comparision of different criteria for the diagnosis of primary myelofibrosis reveals limited clinical utility for measurement of serum lactate dehydrogenese. Hematologica 2010 Nov; 95(11): 1960-1963

13. Hattori N, Fukuchi K, Nakashima H, Maeda T, Adachi D, Saito B et al. Megakaryocyte and platelet unction in polycythemia vera and essential thrombocythemia patients with JAK2 V617F mutation. Int J Hematol 2008; 88:181-188

14. Michiels JJ, Kutti J, Stark P, Bazzan M, Gugliotta L, Marchioli R et al. Diagnosis, pathogenesis and treatment of the myeloproliferative disorders essential thrombocythemia, polycythemia vera and essential megakaryocytic granulocytic metaplasia and myelofibrosis. Neth J Med 1999; 54(2):46-62

15. Michiels J, Thiele J. Clinical and pathological criteria for the diagnosis of essential thrombocythemia, polycythemia vera, and idiopathic myelofibrosis (agnogenic myeloid metaplasia). Int J Hematol 2002; 76(2):133-45

16. Mesa RA, Silverstein MN, Jacobsen SJ, Wollan PC and Tefferi A. Population based incidence and survival figures in essential thrombocythemia and agnogenic myeloid metaplasia: An Olmsted county study, 1976-1995. Am J Hematol 1999;61:10-5

17. Vytrva N, Stacher E, Regitnig P, Zinke-Cerwenka W, Hojas S, Hubmann E et al. Megakaryocytic Morphology and Clinical Parameters in Essential Thrombocythemia, Polycythemia Vera, and Primary Myelofibrosis With and Without JAK2 V617F. Arch Pathol Lab Med 2014; 138;1203-09

18. Marchiolli R, Finazzi G, Landolfi R, Kutti J, Gisslinger $\mathrm{H}$, Patrono $\mathrm{C}$ et al. Vascular and neoplastic risk in a large cohort of patients with polycythemia vera. J Clin Oncol 2005;23:2224-32 
19. Thiele J, Kvasnicka HM, Zankovich R, Diehl V. Relevance of bone marrow features in the differential diagnosis between essential thrombocythemia and early stage idiopathic myelofibrosis. Haematologica 2000; 85(11):1126-34

20. Lan HF, Fang ZH, Zhang Y, Wang XY, Xue F, Zhang L et al. Clinical analysis of 438 patients with essential thrombocythemia. Zhonghua Xue Ye XUE Za Zhi 2008; 29(9):587-91

21. Thiele J, Kvasnicka HM, Zankovich R and Diehl V. Earlystage idiopathic (primary) Myelofibrosis - current issues of diagnostic features. Leuk Lymphoma 2002; 43(5):1035-41

22. Anastasi J, Vardiman JW. Chronic myelogenous leukemia and the Chronic myeloproliferative diseases. In: Knowles DM, editor. Neoplastic Hematopathology. 3rd ed. Philadelphia: Lippincott Williams and Wilkins; 2014. p. 1108-1136

23. Lichtman MA, Tefferi A. Primary Myelofibrosis. In: Kaushansky K, Lichtman MA, Beutler E, Kipps TJ, Seligsohn U, Prachal JT, editor. Williams Hematology. 8th ed. China: McGraw-Hill; 2010. p. 1381-1402

24. Holme S, Simmonds M, Ballek R, Murphy S. Comparative measurements of platelet size by coulter counter, microscopy of blood smears, and light transmission studies. J Lab Clin Med 1981;97:610-22

25. Small BM, Bettigole RE. Diagnosis of myeloproliferative disease by analysis of platelet volume distribution. Am J Clin Pathol 1981;76:685-91

26. Holme S, Murphy S. Studies on the platelet density abnormality in myeloproliferative disease. J Lab Clin Med 1984;103:373-83

27. Rudzki Z, Kawa R, Okon K, Szczygiel E, Stachura J. Objective, planimetry-based assessment of MKs histological pictures in Philadelphia-chromosome-negative chronic myeloproliferative disorders: a perspective for a valuable adjunct diagnostic tool. Virchows Archiv 2005; 448(1):59-67

28. Gianelli U, Lurlo A, Vener C. The significance of bone marrow biopsy and JAK2 V617F mutation in the differential diagnosis between the "early" prepolycythemic phase of polycythemia vera and essential thrombocythemia. Am J Clin Pathol 2008; 130(3):336-342

29. Gianelli U, Vener C, Rafaniello Raviele P, Alessia Moro, Savi F, Annaloro C et al. Essential thrombocythemia or chronic idiopathic myelofibrosis? A single center study based on hematopoietic bone marrow histology. Leuk Lymphoma 2006; 47(9):1774-81

*Corresponding author:

Dr Sharada Rai, Postal Address: Kasturba Medical college, Mangalore, (Manipal Academy of Higher education), Department of Pathology

Lighthouse hill Road, Mangalore , 975001., Karnataka, India

Phone: +91 9740730410

Email: sharada.rai@manipal.edu,sharadarai@yahoo.com

Financial or other Competing Interests: None. 